\title{
Association of increased lipid peroxide levels in the aorta in comparison to the pulmonary artery with the presence of coronary artery disease
}

\author{
ABDUL RAUOOF MALIK ${ }^{1,2}$ \\ ${ }^{1}$ Department of Cardiology, Sher-I-Kashmir Institute of Medical Sciences, Srinagar, Jammu and Kashmir 190011, \\ India; ${ }^{2}$ Department of Medicine, College of Medicine, King Khalid University, \\ Aseer Central Hospital, Abha 61421, Kingdom of Saudi Arabia
}

Received December 15, 2015; Accepted February 17, 2016

DOI: $10.3892 /$ br.2016.614

\begin{abstract}
Atherosclerosis predominantly affects systemic arteries as compared to pulmonary arteries; however, the reasons for this differential predilection are not clear. Oxidative damage caused by free radicals is a key initiating event in atherogenesis and the lungs are able to produce large quantities of free radicals even under physiological conditions. The present study investigated whether pulmonary venous blood reaching the aorta contained greater quantities of lipid peroxides, a marker of oxidative stress, compared to the pulmonary artery. Aortic and pulmonary artery blood samples were collected at the time of cardiac catheterization from 45 consecutive patients ( $38 \%$ female) without acute coronary event and free of other medical disorders, who were scheduled to undergo coronary angiography for anginal chest pain. Lipid peroxides were measured in terms of malondialdehyde (MDA). MDA levels were significantly higher in the aorta compared to the pulmonary artery $(4.93 \pm 1.97$ vs. $3.36 \pm 1.14 \mathrm{nmol} / \mathrm{ml}$; $\mathrm{P}<0.001)$; the difference was significant in patients with angiographic coronary artery disease $(\mathrm{CAD})(\mathrm{P}<0.001)$ compared to the patients without $\mathrm{CAD}(\mathrm{P}=0.071)$. Higher aortic MDA levels were associated with the presence of CAD even following adjustment for major risk factors. The results of the present study demonstrate that aortic blood contains significantly greater levels of lipid peroxides compared to pulmonary artery. This differential oxidative stress between systemic and pulmonary arteries could provide a mechanistic explanation for their difference in the propensity to develop atherosclerosis.
\end{abstract}

Correspondence to: Dr Abdul Rauoof Malik, Department of Medicine, College of Medicine, King Khalid University, Aseer Central Hospital, P.O. Box 641, Abha 61421, Kingdom of Saudi Arabia

E-mail: armalik179@gmail.com

Key words: atherosclerosis, aorta, pulmonary artery, oxidant stress, free radicals, lipid peroxidation

\section{Introduction}

Atherosclerosis typically affects the arterial branch points, bifurcations and tortuous segments of medium-sized and large arteries (1). While the aorta and its major branches, including the coronary, carotid and iliac arteries, constitute the classical sites of atherosclerotic lesions, several other arterial beds are also frequently affected. A notable exception to atherosclerotic involvement exists in the pulmonary circulation. Studies of atherosclerosis of pulmonary arteries are anecdotal, although not unknown (2-5). Traditionally, this higher susceptibility of systemic arteries to atherogenesis relative to pulmonary arteries has been attributed to higher hydrodynamic forces in the former $(6,7)$. When pulmonary arterial pressure is elevated, complex angiopathic lesions develop in the pulmonary arteries that have sometimes been found to resemble systemic atherosclerosis $(5,8,9)$. However, the arterial lesions in pulmonary hypertension are very different from atherosclerotic plaques in their distribution and histopathology (9-12). Therefore, the differential predilection of systemic and pulmonary arterial beds to atherogenesis may not be entirely explained on the basis of differences in blood pressures between the two circulations. Several other known major and novel cardiovascular (CV) risk factors have been demonstrated to promote the development of atherosclerosis through complex biological pathways that remain to be completely elucidated (13). Whether and how these factors present globally within the body and specifically predispose systemic arterial beds to atherogenesis without affecting pulmonary arteries is unknown.

Free radicals are highly reactive chemical species that are ubiquitously found in biological systems. The lung tissue is normally enriched with several enzymatic machineries that produce reactive oxygen species (ROS) and other downstream free radicals to participate in numerous local physiological functions including control of vascular tone, vascular cell growth, angiogenesis, regulation of pneumocyte function and inflammation (14-16). Additionally, for oxygenating blood, pulmonary capillaries have to come in close proximity with environmental oxygen, which also makes lung tissue uniquely exposed and vulnerable to environmental challenges, including airborne irritants and infectious agents, further increasing the 
requirement for the existence of efficient ROS-generating mechanisms. All these factors would be expected to result in higher quantities of free radicals leaving the pulmonary circulation, potentially creating a milieu of greater oxidative stress in the systemic arteries compared to pulmonary arteries. The differential oxidative burden between aortic and pulmonary circulations has not been systematically studied.

Increasing evidence indicates that oxidant damage caused by ROS and other free radicals may be a key event in initiating the development of atherosclerosis (17). Several potential pathways exist through which oxidative damage of biological molecules and their physiological properties may initiate a low-grade inflammation within the vessel wall resulting in a myriad of humoral and cellular events that lead to endothelial dysfunction, and ultimately, atherosclerosis $(14,18,19)$. Due to the oxygen-rich ambience in the lungs with local abundance of ROS-generating systems, the blood reaching the aorta from the pulmonary circulation may contain greater amounts of free radicals than the blood in the pulmonary artery. This differential burden of oxidant stress may explain the greater propensity of systemic arteries to develop atherosclerosis compared to pulmonary arteries.

\section{Materials and methods}

Subject recruitment. The study was carried out in the Department of Cardiology, Sher-I-Kashmir Institute of Medical Sciences (Srinagar, Jammu and Kashmir, India) between January 2004 and April 2005. The study was approved by the Institutional Ethics Committee of Sher-I-Kashmir Institute of Medical Sciences. In total, 45 consecutive patients who were scheduled to undergo elective coronary angiography for further evaluation of typical chest pain were selected. Subsequent to obtaining written informed consent, a complete history, including the history of $\mathrm{CV}$ risk factors and prior $\mathrm{CV}$ disease, was obtained from each participant. A history of hypertension, diabetes and dyslipidemia was defined as self-report of physician diagnosis or use of corresponding medications. Smoking was defined as current smoker or former smoker having stopped $<2$ years ago. Available clinical and laboratory data of the patients were reviewed for any relevant information. Patients with acute coronary event, clinical heart failure or other acute illness were excluded. Patients with chronic kidney disease, liver disease, lung disease, connective tissue disorders and other chronic medical conditions were also excluded.

Coronary angiography. Coronary angiography was performed by experienced cardiologists for clinical indications as aforementioned. A separate written informed consent was obtained from each patient for placement of the femoral venous sheath and pulmonary artery catheterization to obtain blood samples. The entire procedure was performed using the percutaneous transfemoral approach. Briefly, under aseptic precautions, femoral arterial and venous access was obtained using the Judkins technique and corresponding sheaths were placed in. Pulmonary artery catheterization was performed using standard balloon-tipped Swan-Ganz or Cournand catheters. For coronary angiography, standard left and right coronary catheters preloaded with 0.035 inch, tapered, movable core J-wire were advanced through the sheath, and coronary angiography was performed in multiple projections. Coronary angiograms were interpreted offline by the cardiologist in the standard clinical format. For the present study, coronary artery disease (CAD) was diagnosed when the coronary angiogram showed $\geq 50 \%$ luminal stenosis of $\geq 1$ epicardial coronary artery or its major branch.

Collection of blood samples. For each patient, $20 \mathrm{ml}$ of blood was drawn from the main pulmonary artery; in 2 patients the blood samples were collected from the right ventricle due to a difficulty in cannulating the pulmonary artery. Following this, another $20 \mathrm{ml}$ of blood was drawn from the diagnostic coronary catheter from the aortic root immediately above the aortic cusps and just before the first engagement of the coronary ostium. The blood samples were placed into anticoagulant tubes containing liquid K2EDTA $(2.5 \mathrm{mg} / \mathrm{ml}$ blood $)$ and immediately centrifuged at $1,500 \mathrm{x} \mathrm{g}$ for $15 \mathrm{~min}$. The plasma obtained was stored at $-80^{\circ} \mathrm{C}$ until analyzed (within 3 months).

Measurement of lipid peroxides. A bioassay for the free radical burden was carried out by measuring the plasma lipid peroxide levels in terms of malondialdehyde (MDA) using the thiobarbituric acid (TBA) reaction as previously described (modified Yagi's method) (20). The test is based on the principle that MDA formed as a breakdown product of lipoperoxides reacts with TBA to form a colored complex. The lipid material is simply heated with TBA at a low $\mathrm{pH}$ and the MDA-(TBA) adduct is formed, a pink chromogen, which is measured by absorbance at or close to $532 \mathrm{~nm}$ or by fluorescence at $553 \mathrm{~nm}$ following extraction, or not, into butan-1-ol.

Briefly, $100 \mu \mathrm{l}$ of plasma was added to $4 \mathrm{ml}$ of $\mathrm{N} / 12 \mathrm{H}_{2} \mathrm{SO}_{4}$ and the contents were vortex-mixed. Phosphotungstic acid $(10 \%, 0.5 \mathrm{ml})$ was subsequently added and the contents were vortexed again. The reaction mixture was cooled to room temperature for $5 \mathrm{~min}$ and centrifuged at $1,500 \mathrm{x} \mathrm{g}$ for $10 \mathrm{~min}$. Freshly prepared TBA reagent $(1 \mathrm{ml})$ was added to the sediment, the contents were vortex-mixed, heated at $95^{\circ} \mathrm{C}$ for $60 \mathrm{~min}$, and finally cooled to room temperature with tap water. Following this, $5 \mathrm{ml}$ of n-butanol was added, the mixture was agitated thoroughly and was subsequently centrifuged at $1,500 \mathrm{x}$ g for $15 \mathrm{~min}$. The absorbance of the supernatant was measured at $532 \mathrm{~nm}$ on a Hitachi 4020 photometer (Hitachi, Tokyo, Japan). The reference curve was constructed using 1,1,3,3,-tetramethoxypropane [malonaldehyde bis (dimethyl acetal)] obtained from Sigma-Aldrich (cat. no. T1642; St. Louis, MO, USA) as the MDA standard and was found linear up to $300 \mathrm{ng} / \mathrm{ml}$.

Data analysis. Statistical Package for Social Sciences version 19.0 for Windows (IBM, Corp., Armonk, NY, USA) was used for data analysis. Descriptive statistics are presented as mean \pm standard deviation or frequency (percentage). Student's t-test, $\chi^{2}$ test and Fisher's exact test were used to compare the continuous and categorical variables as appropriate. Paired t-test was used to compare the MDA levels between the aortic and pulmonary artery blood samples. Correlation of the MDA levels with clinical variables and angiographic data was assessed using Spearman's rank correlation. Logistic regression was performed to further examine the association of the MDA levels with the presence of CAD 
prior to adjustment (model 1) and following sequentially adjusting for age (model 2), for age and gender (model 3), and for age, gender and other CV risk factors (smoking, hypertension, diabetes, dyslipidemia, total cholesterol, low-density lipoprotein cholesterol, high-density lipoprotein cholesterol and triglycerides), one at a time (model 4). $\mathrm{P}<0.05$ was considered to indicate a statistically significant difference.

\section{Results}

Patient characteristics. Two patients had their blood samples hemolyzed and another patient developed acute febrile illness on the day of catheterization. The characteristics of the remaining 42 subjects included for data analysis are shown in Table I. Patients with CAD were older and had a greater burden of the major $\mathrm{CV}$ risk factors and lower average left ventricular function compared to the patients without CAD.

MDA levels. Overall, the MDA levels were significantly higher in the aorta compared to the pulmonary artery $(\mathrm{P}<0.001)$; the difference was significant in patients with CAD $(\mathrm{P}<0.001)$ compared to in patients without CAD $(\mathrm{P}=0.071)$. Patients with CAD had higher MDA levels in the aortic $(\mathrm{P}<0.001)$ and pulmonary artery $(\mathrm{P}=0.02)$ samples compared to patients without CAD (Table II).

MDA levels showed a positive correlation with the major $\mathrm{CV}$ risk factors; the correlation was particularly notable in the case of aortic MDA levels (Table III). Overall, there was a modest positive correlation between aortic and pulmonary artery MDA levels $(\mathrm{r}=0.61, \mathrm{P}<0.001)$.

Logistic regression analyses. In the logistic regression analyses, the aortic MDA level was significantly associated with the presence of CAD in the univariable model. The association was weaker but persisted following adjustment for age, gender and other major CV risk factors (Table IV). The association of pulmonary artery MDA level with CAD was weak and not present in the adjusted models (data not shown). A greater difference between the aortic and pulmonary MDA levels was positively associated with CAD but the association did not exist subsequent to adjusting for the aortic MDA level (data not shown).

\section{Discussion}

Research conducted in the past few decades has shown that oxidative damage of molecules and cells may be a key event during atherogenesis (17). In vivo, oxidative stress can affect
Table I. Clinical characteristics of the subjects with and without CAD.

\begin{tabular}{|c|c|c|}
\hline Characteristics & No CAD (n=14) & $\operatorname{CAD}(n=28)$ \\
\hline Mean age $\pm S D$, years & $57.1 \pm 5.0$ & $65.0 \pm 7.9$ \\
\hline Female, $\mathrm{n}(\%)$ & $8(57.1)$ & $8(28.6)$ \\
\hline Smoker, n (\%) & $2(14.3)$ & $22(78.6)$ \\
\hline Hypertension, n (\%) & $2(14.3)$ & $20(71.4)$ \\
\hline $\begin{array}{l}\text { Hypertension medication, } \\
\text { n (\%) }\end{array}$ & $2(14.3)$ & $11(39.3)$ \\
\hline Diabetes, n (\%) & $1(7.1)$ & $12(42.9)$ \\
\hline Dyslipidemia, n (\%) & $1(7.1)$ & $15(53.6)$ \\
\hline Statin, n (\%) & $1(7.1)$ & $4(14.3)$ \\
\hline $\begin{array}{l}\text { Mean body mass } \\
\text { index } \pm \mathrm{SD}, \mathrm{kg} / \mathrm{m}^{2}\end{array}$ & $26.0 \pm 2.9$ & $25.9 \pm 4.6$ \\
\hline $\begin{array}{l}\text { Mean systolic blood } \\
\text { pressure } \pm \mathrm{SD}, \mathrm{mmHg}\end{array}$ & $136 \pm 10$ & $143 \pm 16$ \\
\hline $\begin{array}{l}\text { Mean diastolic blood } \\
\text { pressure } \pm \mathrm{SD}, \mathrm{mmHg}\end{array}$ & $80 \pm 8$ & $82 \pm 12$ \\
\hline $\begin{array}{l}\text { Mean total cholesterol } \pm \mathrm{SD} \text {, } \\
\mathrm{mg} / \mathrm{dl}^{\mathrm{a}}\end{array}$ & $163 \pm 20$ & $220 \pm 61$ \\
\hline $\begin{array}{l}\text { Mean LDL cholesterol } \pm \mathrm{SD} \text {, } \\
\mathrm{mg} / \mathrm{dl}^{\mathrm{a}}\end{array}$ & $97 \pm 20$ & $139 \pm 45$ \\
\hline $\begin{array}{l}\text { Mean HDL cholesterol } \pm \mathrm{SD} \text {, } \\
\mathrm{mg} / \mathrm{dl}^{\mathrm{a}}\end{array}$ & $41 \pm 13$ & $43 \pm 10$ \\
\hline $\begin{array}{l}\text { Mean triglycerides } \pm \mathrm{SD} \\
\mathrm{mg} / \mathrm{dl}^{\mathrm{a}}\end{array}$ & $127 \pm 31$ & $211 \pm 94$ \\
\hline Mean LVEF \pm SD,$\%$ & $67.6 \pm 3.5$ & $59.0 \pm 9.10$ \\
\hline
\end{tabular}

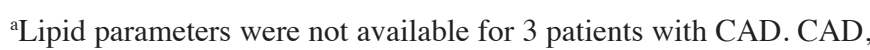
coronary artery disease; SD, standard deviation; LDL, low-density lipoprotein; HDL, high-density lipoprotein; LVEF, left ventricular ejection fraction.

diverse humoral and cellular pathways that initiate and promote the development of atherosclerotic lesions $(18,19,21,22)$. Clinical studies have demonstrated elevated levels of lipid peroxides and other markers of oxidative stress in several atherosclerotic disorders, including CAD, as well as in apparently healthy people with CV risk factors $(23,24)$. Previous studies have suggested that oxidative modification of certain key biomolecules may represent a final common pathway for different $\mathrm{CV}$ risk factors in promoting atherosclerosis (24-27). The finding of elevated MDA levels in patients with CAD compared to those with

Table II. Lipid peroxide levels in the aorta and pulmonary artery.

\begin{tabular}{lccc}
\hline MDA & All subjects & No CAD, n=14 & CAD, n=28 \\
\hline Aortic, nmol/ml & $4.93 \pm 1.97$ & $3.36 \pm 0.93$ & $5.71 \pm 1.88$ \\
Pulmonary, nmol/ml & $3.36 \pm 1.14$ & $2.79 \pm 1.19$ & $3.64 \pm 1.03$ \\
P-value for difference $^{\mathrm{a}}$ & $<0.001$ & 0.071 & $<0.001$ \\
\hline
\end{tabular}

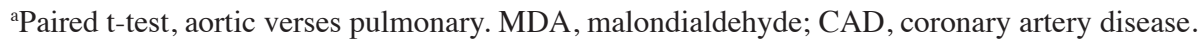


Table III. Correlation of the lipid peroxides with the clinical characteristics.

\begin{tabular}{lccccc}
\hline & \multicolumn{2}{c}{ MDA aortic } & & \multicolumn{2}{c}{ MDA pulmonary } \\
\cline { 2 - 3 } \cline { 6 - 7 } Parameter & Rho & P-value & & Rho & P-value \\
\cline { 7 - 7 } Age & 0.35 & 0.022 & & 0.12 & 0.44 \\
Female & -0.31 & 0.047 & & -0.29 & 0.062 \\
BMI & -0.02 & 0.91 & & -0.03 & 0.84 \\
Smoking & 0.42 & 0.006 & & 0.27 & 0.086 \\
Hypertension & 0.43 & 0.004 & & 0.20 & 0.20 \\
Diabetes & 0.54 & $<0.001$ & & 0.26 & 0.092 \\
Dyslipidemia & 0.39 & 0.016 & & 0.64 & $<0.001$ \\
SBP & -0.01 & 0.96 & & 0.18 & 0.26 \\
DBP & 0.26 & 0.098 & & 0.14 & 0.38 \\
Total cholesterol & 0.46 & 0.003 & & 0.55 & $<0.001$ \\
LDL cholesterol & 0.40 & 0.011 & & 0.47 & 0.003 \\
HDL cholesterol & 0.02 & 0.91 & & -0.23 & 0.048 \\
Triglycerides & 0.46 & 0.003 & & 0.44 & 0.006 \\
LVEF & -0.26 & 0.10 & -0.34 & 0.027 \\
RWMA & 0.35 & 0.024 & 0.24 & 0.12 \\
\hline
\end{tabular}

MDA, malondialdehyde; BMI, body mass index; SBP, systolic blood pressure; DBP, diastolic blood pressure; LDL, low-density lipoprotein; HDL, high-density lipoprotein; LVEF, left ventricular ejection fraction; RWMA, regional wall motion abnormality.

normal coronary arteries in the present study, even following adjustment for $\mathrm{CV}$ risk factors, is thus consistent with the oxidative modification hypothesis of atherosclerosis.
However, the notable finding in the present study is the demonstration of significantly greater levels of MDA in the systemic circulation as compared to the pulmonary circulation in adult individuals with and without angiographic CAD, but particularly in the former group. To the best of our knowledge, this is the first demonstration of the differential oxidative burden between systemic and pulmonary circulations in the context of clinical CAD. Whether the differential oxidant stress between pulmonary and systemic arteries could explain the differences in propensity between these arterial segments to develop atherosclerosis remains to be elucidated.

Due to the global nature of the known major and minor $\mathrm{CV}$ risk factors, pulmonary arteries should, theoretically, at least be as much predisposed to develop atherosclerosis as the systemic arteries. However, atherosclerotic lesions rarely develop in the pulmonary arteries even in the presence of multiple $\mathrm{CV}$ risk factors. The higher blood pressure in the systemic arteries is currently the most widely accepted explanation for their preferential predisposition to atherosclerotic lesion development in comparison to pulmonary arteries $(6,7)$. The 'pressure hypothesis' is also supported by the findings of pulmonary arterial lesions in the presence of pulmonary hypertension $(5,8)$. Certain studies have reported lesions reminiscent of atherosclerotic plaques in the pulmonary arteries of patients with pulmonary hypertension $(5,8,9,28)$. However, systemic atherosclerosis frequently affects people with normal blood pressure, indicating the involvement of factors other than high blood pressure in the etiopathogenesis. Furthermore, the complex angiopathic lesions found in pulmonary hypertension are markedly different from atherosclerotic plaques. For example, the lesions of hypertensive pulmonary arteriopathy typically involve the small pulmonary arteries and arterioles rather than the large and medium-sized arteries affected in

Table IV. Logistic regression showing the association of aortic lipid peroxide levels with the presence of angiographic coronary artery disease.

\begin{tabular}{|c|c|c|c|c|c|c|c|c|}
\hline \multirow[b]{2}{*}{ Parameter } & \multicolumn{2}{|c|}{ Model 1} & \multicolumn{2}{|c|}{ Model 2} & \multicolumn{2}{|c|}{ Model 3} & \multicolumn{2}{|c|}{ Model 4} \\
\hline & $\beta \pm \mathrm{SE}$ & P-value & $\beta \pm \mathrm{SE}$ & P-value & $\beta \pm \mathrm{SE}$ & P-value & $\beta \pm \mathrm{SE}$ & P-value \\
\hline MDA & $1.35 \pm 0.42$ & 0.001 & $1.19 \pm 0.45$ & 0.008 & $1.30 \pm 0.53$ & 0.014 & $1.35 \pm 0.61$ & 0.027 \\
\hline Age & & & $0.17 \pm 0.08$ & 0.039 & $0.19 \pm 0.09$ & 0.028 & $0.20 \pm 0.11$ & 0.085 \\
\hline Male & & & & & $1.61 \pm 1.14$ & 0.16 & $0.90 \pm 1.37$ & 0.51 \\
\hline Smoker & & & & & & & $2.94 \pm 1.40$ & 0.036 \\
\hline Risk factor in the Model & & & & & & & \multicolumn{2}{|c|}{ MDA estimate } \\
\hline Hypertension & & & & & & & $1.16 \pm 0.56$ & 0.038 \\
\hline Diabetes & & & & & & & $1.25 \pm 0.59$ & 0.035 \\
\hline Dyslipidemia & & & & & & & $1.12 \pm 0.53$ & 0.036 \\
\hline Total cholesterol & & & & & & & $0.95 \pm 0.49$ & 0.05 \\
\hline LDL cholesterol & & & & & & & $0.99 \pm 0.48$ & 0.04 \\
\hline HDL cholesterol & & & & & & & $1.19 \pm 0.52$ & 0.021 \\
\hline Triglycerides & & & & & & & $0.95 \pm 0.50$ & 0.058 \\
\hline
\end{tabular}

MDA, malondialdehyde; LDL, low-density lipoprotein; HDL, high-density lipoprotein; SE, standard error; model 1, unadjusted; model 2 , adjusted for age; model 3, adjusted for age and gender; model 4 , adjusted for age, gender and other cardiovascular risk factors, one at a time. The full model 4 for smoking is shown; for models including other risk factors, the estimates and P-values shown are for aortic MDA in the corresponding model. 
systemic atherosclerosis. Additionally, the fibrinoid-proliferative lesions of the hypertensive pulmonary arteriopathy are histopathologically different from the lipid-rich atheromas in systemic arteries (9-12). Therefore, differences in arterial pressure alone do not explain the different atherogenic tendencies between systemic and pulmonary arteries. Studies in laboratory animals have suggested differences in cholesterol handling between aortic and pulmonary arterial tissues; however, the mechanisms for such differences and their relevance to human atherosclerosis remain to be elucidated (29).

The lungs oxygenate the blood and this not only necessitates proximate contact with environmental oxygen, but also exposes the lung tissue to airborne irritants and infectious agents, fighting which requires local generation of ROS. These factors cause the high level of ROS generation in the lung tissue. Furthermore, ROS have vital roles in the local regulation of vascular tone, vascular cell growth, angiogenesis, pneumocyte function and other physiological functions in the lungs. Therefore, the lung tissue is richly endowed with ROS-generating enzymatic machineries (14-16). The pulmonary endothelium is also the known major location of angiotensin-converting enzyme that produces angiotensin II, which is known to have potent effects in augmenting oxidative stress within biological systems (30). Whether angiotensin II could have paracrine effects relevant to oxidative stress within pulmonary vasculature remains to be elucidated. It is possible that the aforementioned and possibly other biological properties specific to pulmonary vasculature could result in a greater load of free radicals leaving the pulmonary vessels, potentially creating greater oxidant stress in the systemic arteries compared to the pulmonary arteries. The observation of significantly higher levels of MDA in the aorta compared to the pulmonary artery in the present study supports this contention. The excess free radicals entering the aorta could potentially create a milieu of greater oxidant stress, reducing the antioxidant defenses in the systemic arteries. It is also possible that the high levels of oxidant stress in the blood entering the left side of the heart would have the most deleterious consequences in the proximal-most segments of the systemic arterial tree prior to antioxidant defenses mitigating their effect. Notably, atherosclerotic lesions are known to be preferentially located within the proximal systemic arteries, such as the aorta and the coronary and carotid arteries. However, these are also the sites most exposed to undergo pulsatile hemodynamic stresses and the central aortic pressure. Whether and to what extent does the elevated oxidant burden in systemic arteries compared to pulmonary arteries explain the known predilection of the former to atherosclerosis requires further exploration.

Furthermore, from the explanation aforementioned, even though a differentially higher oxidant stress in the aorta compared to the pulmonary artery would be physiologically expected, why certain individuals would be more affected by this differential oxidant burden to go on to develop atherosclerosis in systemic arteries is not clear. Deficient antioxidant defenses, exaggerated generation of ROS in the pulmonary capillaries, or interaction between oxidative processes and other vascular stressors could be potential mechanistic explanations that require further exploration.

It is also possible that the increased MDA levels in the aorta compared to the pulmonary artery noted in the present study may be the effect, rather than the cause of systemic atherosclerotic lesions, which are rich in lipids and lipoperoxides. However, any such contribution to aortic MDA levels would be unlikely in blood samples drawn from the aortic root upstream to the usual location of the atherosclerotic lesions. Furthermore, although not statistically significant, MDA levels were higher in the aorta compared to the pulmonary artery even in patients without angiographic CAD, suggesting the higher aortic levels were not likely to be due to atherosclerotic lesions per se.

The principal limitation of the present study is the small sample size, which limits the ability for more thorough statistical analyses. Regression analyses were performed by entering the major $\mathrm{CV}$ risk predictors one at a time as co-variates in testing the association of MDA with CAD. Quantification of oxidant stress in complex biological matrices, particularly its temporal and spatial variations, is challenging and measurement of lipid peroxides may be a suboptimal, albeit well-established, way to assess oxidative damage. For operational reasons, the TBA reaction was used to assay the plasma lipid peroxides, although mass spectrometry and high-performance liquid chromatography are considered more specific in this regard. For the present study, CAD was defined as $\geq 50 \%$ luminal narrowing of epicardial coronary arteries, which precludes investigation of any association of free radical levels with less severe (but potentially important) lesions and subclinical atherosclerosis. Finally, although aortic lipid peroxide levels were higher compared to pulmonary arterial levels, a cause-and-effect association cannot be established from this observational study. Therefore, these findings should be treated as hypothesis-generating and require confirmation in larger studies using more accurate quantification of the oxidative burden in systemic and pulmonary arteries.

In conclusion, the differential burden of lipid peroxides in the aorta compared to the pulmonary artery noted in the present study could potentially provide a mechanistic explanation for the greater propensity of systemic arteries to develop atherosclerosis compared to pulmonary arteries. However, due to the complexity of mechanisms and pathways involved in atherogenesis and its clinical presentation, a direct cause-and effect inference cannot be drawn from these observations. The findings of the present study should only be treated as hypothesis-generating and require confirmation in larger studies.

\section{References}

1. DeBakey ME, Lawrie GM and Glaeser DH: Patterns of atherosclerosis and their surgical significance. Ann Surg 201: 115-131, 1985.

2. Heath D, Wood EH, Dushane JW and Edwards JE: The relation of age and blood pressure to atheroma in the pulmonary arteries and thoracic aorta in congenital heart disease. Lab Invest 9: 259-272, 1960.

3. Botney MD, Kaiser LR, Cooper JD, Mecham RP, Parghi D, Roby J and Parks WC: Extracellular matrix protein gene expression in atherosclerotic hypertensive pulmonary arteries. Am J Pathol 140: 357-364, 1992.

4. Cicconi M, Bonsignore A, Orcioni GF and Ventura F: Primary pulmonary arteries atherosclerosis: Discovering an unusual cause of death in forensic practice. Rom J Leg Med 20: 177-180, 2012.

5. Kravitz KD, Scharf GR and Chandrasekaran K: In vivo diagnosis of pulmonary atherosclerosis. Role of intravascular ultrasound. Chest 106: 632-634, 1994. 
6. Chobanian AV: The influence of hypertension and other hemodynamic factors in atherogenesis. Prog Cardiovasc Dis 26: 177-196, 1983.

7. Nerem RM: Vascular fluid mechanics, the arterial wall, and atherosclerosis. J Biomech Eng 114: 274-282, 1992.

8. Anderson TJ, Larsen ET, Wyse DG and Lester WM: Primary pulmonary hypertension for 30 years. Am J Cardiol 68: 284-285, 1991.

9. Pietra GG: The histopathology of primary pulmonary hypertension. In: The pulmonary circulation. Normal and abnormal Fishman AP (ed). University of Pennsylvania Press, Philadelphia, PA, pp459-472, 1990.

10. Pietra GG, Edwards WD, Kay JM, Rich S, Kernis J, Schloo B, Ayres SM, Bergofsky EH, Brundage BH and Detre KM: Histopathology of primary pulmonary hypertension. A qualitative and quantitative study of pulmonary blood vessels from 58 patients in the National Heart, Lung, and Blood Institute, Primary Pulmonary Hypertension Registry. Circulation 80 1198-1206, 1989.

11. O'Neal RM and Thomas WA: The role of pulmonary hypertension and thromboembolism in the production of pulmonary arteriosclerosis. Circulation 12: 370-382, 1955.

12. Jonigk D, Golpon H, Bockmeyer CL, Maegel L, Hoeper MM, Gottlieb J, Nickel N, Hussein K, Maus U, Lehmann U, et al: Plexiform lesions in pulmonary arterial hypertension composition, architecture, and microenvironment. Am J Pathol 179: 167-179, 2011.

13. Ross R: The pathogenesis of atherosclerosis: A perspective for the 1990s. Nature 362: 801-809, 1993.

14. Al Ghouleh I, Khoo NK, Knaus UG, Griendling KK, Touyz RM, Thannickal VJ, Barchowsky A, Nauseef WM, Kelley EE, Bauer PM, et al: Oxidases and peroxidases in cardiovascular and lung disease: New concepts in reactive oxygen species signaling. Free Radic Biol Med 51: 1271-1288, 2011.

15. van der Vliet A: NADPH oxidases in lung biology and pathology: Host defense enzymes, and more. Free Radic Biol Med 44: 938-955, 2008.

16. Frazziano G, Champion $\mathrm{HC}$ and Pagano PJ: NADPH oxidase-derived ROS and the regulation of pulmonary vessel tone. Am J Physiol Heart Circ Physiol 302: H2166-H2177, 2012.

17. Ross R: Atherosclerosis - an inflammatory disease. N Engl J Med 340: 115-126, 1999.

18. Schächinger V and Zeiher AM: Atherogenesis - recent insights into basic mechanisms and their clinical impact. Nephrol Dial Transplant 17: 2055-2064, 2002.
19. Hennig B and Chow CK: Lipid peroxidation and endothelial cell injury: Implications in atherosclerosis. Free Radic Biol Med 4: 99-106, 1988

20. Iqbal K, Rauoof MA, Mir MM, Tramboo NA, Malik JA, Naikoo BA, Dar MA, Masoodi SR and Khan AR: Lipid peroxidation during acute coronary syndromes and its intensification at the time of myocardial ischemia reperfusion. Am J Cardiol 89: 334-337, 2002

21. Rodríguez-Martínez MA, Martínez-Orgado J, Salaices M and Marín J: Impairment of acetylcholine relaxations by malondialdehyde, a marker of lipid peroxidation. J Vasc Res 33: 463-470, 1996.

22. Nedeljkovic ZS, Gokce $\mathrm{N}$ and Loscalzo J: Mechanisms of oxidative stress and vascular dysfunction. Postgrad Med J 79: 195-199, quiz 198-200, 2003.

23. Stringer MD, Görög PG, Freeman A and Kakkar VV: Lipid peroxides and atherosclerosis. BMJ 298: 281-284, 1989.

24. Rumley AG, Woodward M, Rumley A, Rumley J and Lowe GDO: Plasma lipid peroxides: Relationships to cardiovascular risk factors and prevalent cardiovascular disease. QJM 97: 809-816, 2004.

25. Mullarkey CJ, Edelstein D and Brownlee M: Free radical generation by early glycation products: A mechanism for accelerated atherogenesis in diabetes. Biochem Biophys Res Commun 173: 932-939, 1990.

26. Redón J, Oliva MR, Tormos C, Giner V, Chaves J, Iradi A and Sáez GT: Antioxidant activities and oxidative stress byproducts in human hypertension. Hypertension 41: 1096-1101, 2003.

27. Alexander RW: Theodore Cooper Memorial Lecture. Hypertension and the pathogenesis of atherosclerosis. Oxidative stress and the mediation of arterial inflammatory response: A new perspective. Hypertension 25: 155-161, 1995.

28. Brinkman GL: Ultrastructure of atherosclerosis in the human pulmonary artery. Am Rev Respir Dis 105: 351-357, 1972.

29. Schwenke DC: Comparison of aorta and pulmonary artery: I. Early cholesterol accumulation and relative susceptibility to atheromatous lesions. Circ Res 81: 338-345, 1997.

30. Nickenig G and Harrison DG: The AT(1)-type angiotensin receptor in oxidative stress and atherogenesis: Part II: AT(1) receptor regulation. Circulation 105: 530-536, 2002. 